\title{
RELATO DE EXPERIÊNCIA DE EXTENSÃO NO CUIDADO DA SAÚDE DE FAMILIARES, CUIDADORES E INDIVÍDUOS COM SÍNDROME DE DOWN
}

Isabel Cristina Gavazzoni Bandeira Andrade Universidade Regional de Blumenau iandradegb@gmail.com

Thais França

Universidade Regional de Blumenau thaisf05@gmail.com

Letícia Salm

Universidade Regional de Blumenau leticiasalm@yahoo.com

Ana Marize Pacheco de Souza Universidade Regional de Blumenau ama.souza@terra.com.br

Hamilton Fogaça

Universidade Regional de Blumenau h.fogaca@terra.com.br

Maria Cláudia Schmitt Lobe Universidade Regional de Blumenau maria.1@terra.com.br

Laís Dadan Perini

Universidade Regional de Blumenau lais_dadan@hotmail.com.br

Ana Bruna Schmitt Universidade Regional de Blumenau bru.schmitt@yahoo.com.br

Pollyanna Mayara da Silva Universidade Regional de Blumenau pollyannams@hotmail.com

\section{Resumo}

Apresentar ações desenvolvidas por cursos da área da saúde da Universidade Regional de Blumenau (FURB), a saber medicina, odontologia e psicologia, em um programa de extensão, de fevereiro de 2015 até o período atual, e o retorno dado pela comunidade que frequenta a Associação Sorrir para Down em Blumenau, frente às ações desenvolvidas. As atividades foram planejadas de acordo com as demandas oriundas da associação, e aconteceram sob a forma de palestras sobre saúde mental, bucal e geral, oficinas, teatros e rodas de conversas. A atuação das diferentes áreas possibilitou um convívio grupal, superação de desafios e construção do conhecimento. A aproximação entre acadêmicos da Universidade com a comunidade proporcionou o estabelecimentos de vínculos, a troca de experiências, a prestação de informação ao grupo atendido e encaminhamentos à assistência especializada, quando necessário. Além de contribuir para a conscientização e melhora na saúde da pessoas envolvidas, segundo relatos da comunidade.

Palavras-chave: Extensão. Saúde. Síndrome de Down.

\section{REPORT EXTENSION EXPERIENCE IN THE HEALTH CARE OF FAMILY, CAREGIVERS AND INDIVIDUALS WITH DOWN'S SYNDROME}

\section{Abstract}

To present actions developed by courses of the health area at the Universidade Regional de Blumenau (FURB), as medicine, dentistry and psychology, in an outreach program from February of 2015 to the present period, and the feedback given by the community that attends the Associação Sorrir para Down in Blumenau, in front of the developed actions. The activities were planned according to the requests of the association, and happened in form of lectures on mental, oral and general health, theater activities, workshops and conversation. The involvement of the different areas enabled a group life, overcome challenges, socializes and built knowledge. The approach between academics from different University courses and the community with special needs enabled the establishment of links, the exchange of experiences, the provision of information to the group attended and referrals to specialized assistance, when necessary. Besides contributing to the awareness and improvement in the health of the people involved, according to community reports.

Key words: Extension. Health. Down's Syndrome.

\section{INFORME DE EXPERIENCIA EN EXTENSIÓN EN EL CUIDADO DE LA SALUD DE FAMILIARS, CUIDADORES Y PERSONAS CON EL SÍNDROME DE DOWN}

\section{Resumen}

Acciones presentes desarrollados por los cursos del área de salud de la Universidad Regional de Blumenau (FURB), a saber, la medicina, la odontología y psicología, en un programa de extensión del mes de febrero de 2015 para el período actual, y el retorno de la comunidad que frequenta la Associación Sonreir para Down en Blumenau, en comparación con las acciones desarrolladas. Las actividades fueron planificadas de acuerdo a las demandas de la asociación, y se llevaron a cabo en forma de conferencias sobre ruedas de salud, talleres, teatro y conversaciones mentales, orales y generales. El rendimiento de las distintas zonas de activar una vida de grupo, la superación de los desafíos y la construcción del conocimiento. La aproximación entre estudiantes de la Universidad con esta comunidad proporcionan los establecimientos de vínculos, intercambio de experiencias, proporcionando información para el grupo tratado y referidos a la atención especializada cuando sea necesario. Además de contribuir a la sensibilización y la mejora en la salud de las personas involucradas, de acuerdo con informes de la comunidad.

Palavras clave: Extensión. Salud. Sindrome de Down. 
Relato de experiência de extensão no cuidado da saúde de familiares, cuidadores e indivíduos com Síndrome de Down

\section{INTRODUÇÃO}

A Síndrome de Down (SD) é uma cromossomopatia causada pela trissomia do 21 (ASIM et al., 2015), tendo como características alterações morfofuncionais com aparentes características físicas e aprendizagem mais lenta (TORQUATO et al., 2013). Acredita-se que o Brasil tenha cerca de 300.000 pessoas com SD (PRADO et al., 2009), sendo que a incidência aumenta com a idade materna avançada.

As manifestações clínicas da SD incluem atraso no desenvolvimento motor, hipotonia muscular generalizada, frouxidão ligamentar, deficiência mental, problemas de audição e visão, e cardiopatias congênitas (TECKLIN, 2002). Em geral, os indivíduos com SD apresentam um atraso na aquisição de marcos motores (RODRIGUES et al., 2011).

É de extrema importância a qualidade da interação entre pais e filhos, assim aspectos importantes como a fala, as emoções e o desenvolvimento das áreas cognitivas acontecem de forma mais adequada (VOIVODIC; STORER, 2002). Essa qualidade de interação está relacionada com o desenvolvimento da criança com SD nos primeiros anos de vida, já que o estímulo deve ser feito precocemente, e juntamente com a família. Quando bem estimulada, as crianças com SD podem ter uma maior independência e participar de atividade em grupo em todos os aspectos, mas dentro de suas limitações (MARI, 2000; SANTOS, PINTO, 1993). O prognóstico do paciente com SD depende em grande parte da intervenção precoce multidisciplinar e da estimulação proporcionada pelo meio ambiente, que oriente o indivíduo no seu desenvolvimento em rumo ao aumento da sua autonomia (COELHO; APEMAC, 2016).

Atualmente tem-se observado ações multidisciplinares na área da saúde que vem exigindo compreensão dos profissionais sobre os fatores biológicos, comportamentais, ambientais e sociais que determinam o processo saúde doença em pessoas com necessidades especiais, incluindo a SD (MOISÉS; WATT, 2000). São considerados pacientes com necessidades especiais pessoas com doenças ou condições que exigem atendimento diferenciado (SANTOS; SABBAGH-HADDA, 2003). Os profissionais da saúde ainda não estão capacitados a atendê-los, pois na prática diária pouca atenção é dedicada a eles, devido à baixa demanda (MARCHIONI, 2002).

As diversas transformações que tem acontecido nos últimos tempos, sejam social, política ou sanitária, estabelecem aos profissionais de saúde novos desafios para a prática e para sua formação (VASCONCELOS; PEREIRA; CRUZ, 2008). O perfil pretendido para esses profissionais requer a formação humanista, crítica, generalista e reflexiva para atuar em todos os níveis de atenção à saúde, com base no rigor técnico e científico. Com essas considerações, a 
Relato de experiência de extensão no cuidado da saúde de familiares, cuidadores e indivíduos com Síndrome de Down

extensão universitária desempenha um papel muito importante na formação de profissionais, realizando a ligação da Universidade com a sociedade (HENNINGTON, 2005).

Para que esta ligação aconteça é importante um programa com integração de diferentes cursos voltado para pessoas com SD e seus familiares. O programa intitulado "O cuidado integral da saúde de familiares/cuidadores e indivíduos com Síndrome de Down", que está em desenvolvimento desde fevereiro de 2015 até o período atual, envolve acadêmicos e docentes dos cursos de medicina, odontologia e psicologia, e visa desenvolver a capacidade de trabalhar em equipe de forma interdisciplinar, aprimorar as aptidões individuais e a inserção do acadêmico na comunidade, estudando e desenvolvendo temas importantes sobre o universo das famílias e pessoas com SD. Os programas de extensão também propiciam campo para desenvolver atividades de pesquisa e ensino.

Sendo assim, cabe pensar na importância de programas de extensão, voltados para promoção de saúde, prevenção de agravos e intervenção. Estes programas devem buscar auxiliar os pais e mães no processo de adaptação à criança com SD, tanto no que diz respeito aos aspectos físicos da síndrome, proporcionando-lhes acesso à informação, e oferecendo-lhes orientações nos cuidados demandados pela criança. Isto permitirá que pais, mães e demais familiares consigam lidar de modo mais adequado com a criança com SD, facilitando o desenvolvimento de todo o seu potencial e contribuindo para a qualidade de vida destas famílias (HENN et al., 2008).

Um aspecto importante para a inclusão social das pessoas com SD é a saúde bucal. Os familiares, os indivíduos com SD e os demais profissionais que acompanham precisam estar cientes da influência da saúde bucal no estado de saúde geral desses indivíduos (KAYE et al., 2005). O estudo de Descamps e Marks (2015) avaliou o conhecimento sobre saúde bucal de pais de crianças com SD, identificado que alguns pais nunca receberam orientações de como proceder à higiene bucal de seus filhos. Várias manifestações orofaciais podem ser encontradas em indivíduos com SD, dentre as principais pode-se incluir macroglossia, respiração bucal, erupção retardada, microdontia, anodontia e má-oclusão (BERTHOLD et al., 2004). A doença periodontal também é comum, por apresentarem um sistema imune falho e dificuldade de fazer a higiene dental sozinhos (CAVALCANTE; PIRES; SCAREL-CAMINAGA, 2009).

Segundo Conill (2004), é relevante considerar o significado que as recomendações de cunho preventivo podem ter na vida das pessoas com SD, pois um problema dentário pode ser evitado caso o paciente receba cuidados preventivos antes da instalação ou agravamento. Estes agravamentos também se estendem para a saúde mental, na medida em que se sabe que estes sujeitos, apresentam, desde a mais tenra infância, atrasos no desenvolvimento psicomotor. Neste 
Relato de experiência de extensão no cuidado da saúde de familiares, cuidadores e indivíduos com Síndrome de Down

aspecto, considerar a dimensão subjetiva dos sujeitos com SD passa a ser objeto de trabalho, nesse projeto, com a inclusão da Psicologia. A Medicina tem como enfoque as ações de prevenção e de promoção da saúde e do bem estar dos familiares dos indivíduos com SD, englobando questões como avaliação do risco de doenças cardiovasculares, obesidade, importância da atividade física e lazer, alimentação saudável, tabagismo, e importância do acompanhamento ginecológico para as mulheres.

Diante do exposto, é importante relatar as ações desenvolvidas pelo programa de extensão "O cuidado integral da saúde de familiares/cuidadores e indivíduos com Síndrome de Down”, que envolvem acadêmicos e docentes dos Cursos de odontologia, medicina e psicologia da Universidade Regional de Blumenau (FURB), e o retorno dado pela comunidade que frequenta a Associação Sorrir para Down em Blumenau, frente às ações desenvolvidas.

\section{MATERIAIS E MÉTODOS}

O programa de extensão "O cuidado integral da saúde de familiares/cuidadores e indivíduos com Síndrome de Down” é constituído por dois projetos nas áreas de Medicina, Odontologia e Psicologia. Participam deste programa acadêmicos extensionistas bolsistas e voluntários, docentes, dirigentes e a comunidade que frequenta a Associação Sorrir para Down, localizada em Blumenau - S.C. As atividades do programa são realizadas na Associação Sorrir para Down, localizada na rua Margarida Waldrich, n 230, bairro Água Verde, em Blumenau, S.C. A Associação é uma ONG que atende cerca de 200 famílias de Blumenau e região, e busca o apoio de profissionais da saúde para realizar atendimento das pessoas com SD.

O programa de extensão teve início em fevereiro de 2015 e a cada início de semestre são realizadas reuniões com docentes e discentes, além de membros da Associação, para elaboração do cronograma e planejamento das atividades.

Todas as atividades foram planejadas de acordo com a demanda vinda da Associação Sorrir para Down, e voltadas para as pessoas com SD e seus responsáveis, através de palestras, oficinas, atividades lúdicas, rodas de conversa e eventos de confraternização. As atividades grupais dialogadas são referentes à temas relacionados a promoção e prevenção de saúde. As pessoas e familiares que apresentam problema de saúde referidos ao grupo nas atividades, foram encaminhados para atendimento específico, seja nos Ambulatórios da Universidade, Postos de Saúde do Município de Blumenau ou Clínicas Odontológicas da Universidade. 
Relato de experiência de extensão no cuidado da saúde de familiares, cuidadores e indivíduos com Síndrome de Down

É através de jogos que o ser humano melhora sua capacidade de raciocínio lógico, interação, criatividade e eleva sua autoestima (MAR, 2006). A partir disso, no primeiro encontro do semestre na Associação Sorrir para Down, ocorreu a socialização entre acadêmicos, docentes e a comunidade presente, utilizando a dinâmica passa-repassa com caixa surpresa e frases que continham adjetivos.

Em outros momentos, realizaram-se palestras com o auxílio de projetor multimídia, nas quais foram abordados temas como as principais doenças da boca, hábitos bucais deletérios, higiene bucal, desenvolvimento da criança com SD e os aspectos psicomotores.

Durante um dos encontros foi realizada uma oficina com o tema análise sensorial com os pais/cuidadores e pessoas com SD, onde utilizaram-se alimentos pegajosos (bolachas e chocolate) e alimentos detergentes (maçã, cenoura e queijo). Após análise sensorial foi abordado a importância de bons hábitos alimentares para a prevenção de doenças. Em seguida, realizou-se instrução de higiene bucal para todos os participantes da oficina, com o auxílio de macromodelos e revelação de biofilme dental utilizando pastilhas evidenciadoras.

Ainda no primeiro semestre de 2015, foram desenvolvidas atividades a partir de rodas de conversa para que houvesse a troca de experiências com pais e cuidadores das pessoas com SD. Nessa roda de conversa, trabalhou-se a expectativa do futuro de seus filhos, através da dinâmica do fio, no qual os participantes da dinâmica tiveram que cortar um pedaço de fio de lã do tamanho que quisessem. Após isso, o participante ia enrolando o fio no dedo e falando da expectativa para o futuro de seus filhos até que o fio estivesse totalmente enrolado no dedo.

Ao longo do programa também realizaram-se atividades lúdicas temáticas sobre saúde voltadas ora para as pessoas com SD, ora para seus responsáveis. Os acadêmicos confeccionaram jogo da memória, jogo de tabuleiro e dominó feito com desenhos ilustrativos sobre saúde, jogo de mitos e verdades com perguntas relacionadas aos temas sobre saúde expostos nas rodas de conversa e palestras, teatros com fantoches temáticos feitos de caixas de leite e E.V.A. Além disso, trabalhos artesanais, como a confecção de porta-escova com toalhas de lancheira e fio de linha para costurá-lo, buscando aumentar o vínculo entre os presentes.

As avaliações foram realizadas no final de cada atividade executada, através de diálogo com os participantes. A cada encontro foi solicitado aos acadêmicos um relatório individual, fazendo parte do processo avaliativo, sobre a experiência vivenciada em cada atividade.

A psicologia desenvolveu a maior parte de suas atividades com os pais e cuidadores, buscando acolhe-los e orientá-los na maneira de lidar com os indivíduos com SD, enfatizando a importância da autonomia e respeitando o potencial de cada um. 
Relato de experiência de extensão no cuidado da saúde de familiares, cuidadores e indivíduos com Síndrome de Down

Já no que diz respeito às atividades realizadas pela medicina voltadas aos cuidadores, os encontros foram realizados na Associação Sorrir para Down. No primeiro, foi feita uma atividade demonstrando a quantidade de açúcar, sal e gordura que existem nos alimentos industrializados que são frequentemente consumidos no dia a dia. $O$ encontro teve como propósito uma conscientização sobre as quantidades de sal, açúcar e gordura existentes nestes alimentos, além de oferecer formas mais naturais de alimentação, a fim de incentivar os cuidadores a praticarem uma alimentação mais saudável com objetivo de melhorar o bem estar e prevenir o ganho excessivo de peso que pode levar a doenças como diabetes e hipertensão arterial.

Outra atividade teve como propósito informar sobre o significado de lazer, pois muitos relataram cansaço, tristeza para atividades diárias que referiram ser devido à sobrecarga no cuidado com os indivíduos com SD. Mostrou-se, então, a importância que o lazer tem no cuidado da saúde mental, tão importante quanto a física, além de ter sido apresentadas ideias de lazer gratuitas disponíveis na cidade de Blumenau - SC.

Foi conversado também sobre a necessidade do cuidado da saúde da mulher, uma vez que a grande maioria dos cuidadores que frequentam a associação são mulheres. Foi questionado sobre como está a saúde ginecológica das cuidadoras, e com a obtenção das informações foi elaborado uma roda de conversa. Discutiu-se sobre a higiene íntima, exame preventivo, exames de mama, como auto-exame e mamografia e elucidou a importância desta avaliação no diagnóstico precoce e foram informados os locais de atendimento gratuito para a população.

\section{RESULTADOS E ANÁLISES}

O presente programa conseguiu a participação e integração de acadêmicos e docentes dos cursos de odontologia, medicina e psicologia, com uma atuação comprometida com foco na promoção da saúde de pessoas com SD e seus familiares/cuidadores. É importante destacar o esforço do grupo em aproximar, relacionar e integrar os conhecimentos evitando a fragmentação do saber, sem falar na experiência e o convívio grupal adquiridos. A extensão universitária requer, entre outras atitudes, a disposição para atuar de forma cooperativa e com muita responsabilidade social (SÍVERES, 2013).

A atuação dos acadêmicos e professores no programa possibilitou vivenciar uma experiência extra muro, conhecer o universo das pessoas com SD e integrar com a comunidade. A extensão deve ser vista como uma via de mão-dupla, onde há a integração, aproximação, e parceria dos acadêmicos e professores com a sociedade, através da elaboração da prática 
Relato de experiência de extensão no cuidado da saúde de familiares, cuidadores e indivíduos com Síndrome de Down

acadêmica de acordo com a realidade e as necessidades específicas da sociedade, o que é de suma importância para a formação profissional (BARJA-FIDALGO et al., 2014). O contato e a vivência precoce do acadêmico com o mundo real através de atividades extencionistas práticas é essencial para a sua formação, permitindo aplicar os conhecimento adiquiridos em sala de aula no cotidiano (HENNINGTON, 2005).

Estabeleceu-se com o decorrer da execução desse programa vínculos, entre academia e a comunidade, fato que pôde ser comprovado pelo número crescente de participantes nas atividades e o grau de satisfação comentado pelos presentes. Também evidenciou-se a formação deste vínculo entre os acadêmicos, proporcionada pela troca de experiências, traduzida em um crescente envolvimento com as atividades e a comunidade. Com a intenção de atender de maneira mais adequada as necessidades da comunidade, é importante identificar as características particulares dessa população e conhecer sua realidade, elementos essenciais para a aproximação entre comunidade e profissionais da saúde (SOUZA; CARVALHO, 2003).

O processo de retorno dos participantes, através de rodas de conversas, foi muito importante, funcionando como um norteador de como planejar as atividades, o que deve ser mantido e aprimorado. A roda de conversa é mais que disposições circulares de cadeira, é um modo crítico de pensar os papéis socialmente construídos transversalizados pelas históricas e desiguais relações de classe, gênero e etnia (SAMPAIO et al., 2014). Os trabalhos artesanais e rodas de conversas com os responsáveis com temas específicos, onde surgiram narrativas sobre o cotidiano, sobre suas histórias de vida, sobre seus filhos e como foi descobrir que seu bebê "idealizado" tinha SD, resultaram em uma rica troca de experiências entre eles.

As rodas de conversa proporcionam a integração entre os sujeitos e estimulam a comunicação e a partilha de sentimentos, opiniões e discussões (COSTA et al, 2015). As informações dadas provocaram reações entre os participantes, como exemplo os cuidadores se mostraram surpresos com a informação dada sobre a quantidade de sal e açúcar nos alimentos habitualmente utilizados por eles.

No que diz respeito aos cuidadores, sabe-se que a chegada de um indivíduo com SD no meio familiar traz uma série de demandas e desafios. As mães, na maioria das vezes, relataram sentir-se as principais responsáveis pelas crianças e que por vezes, sentem-se sobrecarregadas. Ao tentar desempenhar da melhor forma as múltiplas atividades a elas destinadas, as mulheres referem pouco tempo para seus cuidados pessoais (KORTCHMAR et al., 2014). Ao se investigar melhor a saúde das mães de crianças com $\mathrm{SD}$, estudos revelaram que muitas referiam sensação se fadiga, nervosismo e sintomas depressivos na maior parte do tempo, as mesmas ainda relatam uma percepção ruim sobre sua própria saúde sem perspectiva de melhora (HENN et al., 2008). 
Relato de experiência de extensão no cuidado da saúde de familiares, cuidadores e indivíduos com Síndrome de Down

Uma família que convive diariamente com indivíduos com SD muitas vezes deixa de lado o seu cuidar para dedicar-se integralmente à pessoa acometida pela síndrome. Este deixar-se do autocuidado pode gerar frustração que pode intervir negativamente na necessidade da atenção especial ao indivíduo acometido pela síndrome.

Quanto aos encontros com temas médicos abordados pelas acadêmicas de medicina, pode-se perceber o quão difícil, mesmo com orientações, é para o cuidador de pessoas com SD a percepção da necessidade de olhar um pouco para a própria saúde. Embora sejam mostrados os riscos que levam uma má alimentação, a falta de exercícios físicos, e os benefícios de um acompanhamento médico, exames de rastreamento em dia, muitos ainda deixam de lado cuidados com a própria saúde, porque o resultado é pouco palpável no presente, colocam o bem estar do indivíduo com SD em primeiro lugar. Entretanto conseguiu-se ver algumas mães e cuidadores indo atrás de seus exames de rotina, mostrando que é difícil conseguir fazer com que estes se preocupem com a sua saúde, mas é possível transmitir a importância de manter uma saúde adequada para algumas pessoas. No momento em que a comunidade identifica-se com as mudanças de hábitos, a promoção de uma vida saudável é mais efetiva e duradoura (SOUZA; CARVALHO, 2003).

Durante as atividades demandas foram referidas ao grupo, como por exemplo: exame preventivo de câncer de colo uterino, atendimento odontológico, dentre outros, e foram encaminhados para atendimento específico nos Ambulatórios da Universidade, Postos de Saúde do Município de Blumenau ou Clínicas Odontológicas da Universidade

Em relação à saúde bucal foi visível, através de revelação de biofilme dental, a melhora nos cuidados bucais, envolvendo higiene bucal e hábitos alimentares saudáveis. Muitos pacientes foram incentivados e encaminhados para serem atendidos na Universidade e estão em tratamento. Moimaz et al. (2015) e Sabóia et al. (2014) constataram que a atividade extensionista gera acesso aos serviços odontológicos e esclarece o público-alvo sobre cuidados com a higiene bucal, assim como inova a aprendizagem do acadêmico baseando-se em conhecimento teóricoprático com metodologia de ensino inovadora.

Além das atividades de extensão concomitantes às de ensino, projetos de pesquisa têm surgido à partir das práticas desenvolvidas com a comunidade das pessoas com SD, devido à necessidade de se conhecer a realidade dos participantes da Associação. Como exemplo as pesquisas já concluídas "Diabetes Mellitus tipo I e Tireoidite de Hashimoto em crianças com Síndrome de Down" e "Doenças tireoideana em pacientes gemelares com Síndrome de Down”, e as em desenvolvimento: "Avaliação biológica e bioquímica da saliva em relação a doença cárie: um estudo em pessoas com e sem Síndrome de Down" e "Condição periodontal em nível de 
Relato de experiência de extensão no cuidado da saúde de familiares, cuidadores e indivíduos com Síndrome de Down

higiene bucal de pessoas com Síndrome de Down na faixa etário dos 11 a 17 anos que frequentam a Associação Sorrir para Down de Blumenau/S.C.”. Pesquisa, ensino e extensão articulam-se, na formação acadêmica, de modo a "promover uma consciência profissional eticamente fundamentada e empiricamente atualizada" (MOITA; ANDRADE, 2009).

O programa viabilizou a divulgação das atividades em eventos científicos, como nas mostras integradas de Ensino, Pesquisa e Extensão da FURB, de 2015 e 2016, e durante as semanas acadêmicas dos cursos envolvidos. Também em todas as atividades foram confeccionados e entregues materiais informativos e educativos para familiares/cuidadores e pessoas com SD.

A integração entre ensino-serviço, outro resultado conquistado, tem proporcionado ações voltadas à comunidade, com foco na atenção em saúde, tanto em atividades preventivas com de encaminhamentos para tratamento na Universidade.

Conseguiu-se perceber o impacto do programa na comunidade através do retorno de depoimentos dos participantes cuidadores/familiares e pessoas com SD, abordando aspectos positivos e negativos do programa. Quanto aos depoimentos negativos, estes estão mais relacionados às limitações de horários impostas pelas matrizes curriculares e disponibilidade dos acadêmicos e professores em participar em períodos mais flexíveis. Já os retornos positivos reforçaram a aquisição de novos conhecimentos, o amparo sentido pelos familiares/cuidadores em relação às suas demandas, tanto psicológicas, e/ou de tratamento clínico médico e odontológico.

Por fim, os aspetos positivos foram percebidos através do aumento gradativo da adesão, da participação efetiva em todas as atividades propostas desenvolvidas, e da maior procura por atendimento institucional. Os cuidadores procuram saber sobre novas palestras e atividades do Programa de Extensão na Associação.

\section{CONSIDERAÇÕES FINAIS}

As pessoas com SD apresentam características no seu desenvolvimento que requerem uma atenção diferenciada e ampliada, que envolve tanto aspectos físicos quanto emocionais. Também a família/cuidadores destas pessoas necessitam de apoio nestes mesmos aspectos.

Neste cenário, destaca-se a relevância de um programa de extensão com a participação integrada de vários cursos, possibilitando a socialização e construção do conhecimento, 
Relato de experiência de extensão no cuidado da saúde de familiares, cuidadores e indivíduos com Síndrome de Down

enriquecendo a formação acadêmica, e contribuindo para um desenvolvimento saudável dos familiares/cuidadores e pessoas com SD.

\section{REFERÊNCIAS}

ASIM, A. et al. Down syndrome: an insight of the disease. J Biomed Sci., v.11, n. 22, p.41, 2015.

BARJA-FIDALGO, F. et al. Projeto de extensão crescer sorrindo: integrando ensino, pesquisa e extensão em odontologia por 12 anos crescer sorrindo. Revista Conexão UEPG, v. 10, n. 1, p. 93-101, Jan./Jun. 2014.

BERTHOLD, T.B. et al. Síndrome de Down: aspectos gerais e odontológicos. R. Ci. méd. biol, v. 3, n. 2, p. 252-260, Jun./Dez. 2004.

CAVALCANTE, L.B.; PIRES, J.R.; SCAREL-CAMINAGA, R.M. Doença periodontal em indivíduos com Síndrome de Down: enfoque genético. RGO, v. 57, n. 4, p. 449-453, Out./Dez. 2009 .

COELHO, C.; APEMAC, M. C. A Síndrome de Down. Disponível em:

$<$ http//www.psicologia.pt/artigos/ ver artigo.php>. Acesso em: 11 de outubro de 2016.

CONILL, E. M. Avaliação da integralidade:conferindo sentido para os pactos na programação de metas dos sistemas municipais de saúde. Cad Saúde Pública, v. 20, n. 5, p. 1417-1423, Set./Out. 2004.

COSTA, R. R. O. et al. As rodas de conversa como espaço de cuidado e promoção da saúde. Rev. de Atenção à Saúde, v. 13, n. 43, p. 30-36, Jan./Mar. 2015.

DESCAMPS, I.; MARKS, L. A. Oral health in children with Down syndrome: parents views on dental care in Flanders (Belgium). Eur J Paediatr Dent, v.16, n.2, p. 143-8, 2015.

HENN, C. G.; PICCININI, C. A.; GARCIAS, G. L. A família no contexto da Síndrome de Down: Revisando a literatura. Psicologia em Estudo, v. 13, n. 3, p. 485-493, Jul./Set. 2008.

HENNINGTON, E. A. Acolhimento como prática interdisciplinar num programa de extensão universitária. Cad. Saúde Pública, v. 21, n. 1, p. 256-265, Jan./Fev. 2005.

KAYE, P. L. et al. Views and experiences of parents and siblings of adults with Down Syndrome regarding oral healthcare: a qualitative and quantitative study. Br Dent J, v. 198, n. 9, p. 571-278, Mai. 2005.

KORTCHMAR, E. et al. Vivência da mulher com um filho com síndrome de down em idade escolar. Texto Contexto Enfermagem, v. 23, n. 1, p. 13-20, Jan./Mar. 2014 
Relato de experiência de extensão no cuidado da saúde de familiares, cuidadores e indivíduos com Síndrome de Down

MAR, G. D. O lúdico, o ensino das línguas e os temas transversais. Nuances: estudo sobre educação, v. 13, n. 14, p. 153-165, Jan./Dez. 2006.

MARCHIONI, S. A. E. A formação de vínculo no atendimento odontológico. In: SEGER, L. cols. Psicologia e Odontologia: uma abordagem integradora. $4^{\mathrm{a}}$ ed. São Paulo: Ed. Santos, 2002.

MARI, J. D. Além dos limites. Veja, v. 13, p. 172-173, Abril 2000.

MOIMAZ, S. A. S. et al. Extensão universitária como ferramenta geradora de ensinoaprendizagem e produtora de pesquisa. Revista Conexão UEPG, v. 11, n. 2, p. 140-149, Mai./Ago. 2015.

MOITA, F. M. G. S. C.; ANDRADE, F. C. B. Ensino-pesquisa-extensão: um exercício de indissociabilidade na pós-graduação. Revista Brasileira de Educação, v. 14, n. 41, p. 269- 280, Mai./Ago. 2009.

MOISÉS, S. T.; WATT, R. Promoção de saúde bucal: definições. In: BUISCHI, Y. P. Promoção de saúde bucal na clínica odontológica. São Paulo: Artes Médicas, 2000.

PRADO, M. B. et al. Acompanhamento nutricional de pacientes com Síndrome de Down atendidos em um consultório pediátrico. O Mundo da Saúde, v. 33, n. 3, p. 335-346, Mai. 2009.

RODRIGUES, L. M. et al. Comparação das habilidades motoras em crianças com síndrome de Down e crianças sem distúrbios de desenvolvimento. Brazilian Journal of Motor Behavior, v. 6, n. 1, p. 45-55, 2011.

SABÓIA, V. P. A. et al. Programa odontológico preventivo para gestantes adolescentes - projeto sorridente: relato de experiência. Revista Extensão em Ação, v. 2, n. 7, p.140-152, Jul./Dez. 2014.

SAMPAIO, J. et al. Limites e potencialidades das rodas de conversa no cuidado em saúde: uma experiência com jovens no sertão pernambucano. Revista Interface- Comunicação Saúde e Educação, v. 18, n. 2, p. 1299-1312, 2014.

SANTOS, L. R.; PINTO, V. L. Síndrome de Down - A mãe pode ser uma co-terapeuta, p. 33-54, 1993.

SANTOS, M. T. B. R.; SABBAGH-HADDA, A. Quem são os pacientes com necessidades especiais? In: CARDOSO, J. A.; MACHADO, M. E. L. Odontologia, arte e conhecimento. São Paulo: Artes Médicas, 2003.

SÍVERES, L. A Extensão universitária como um princípio de aprendizagem. Brasília: Liber Livro, 2013, p. 272.

SOUZA, R. A.; CARVALHO, A. M. Programa de Saúde da Família e qualidade de vida: um olhar da Psicologia. Estud. psicol., v. 8, n. 3, p. 515-523, Set./Dez. 2003.

TECKLIN, J. S. Fisioterapia pediátrica. 3. ed. Porto Alegre: Artmed, 2002. 479 p. 
Relato de experiência de extensão no cuidado da saúde de familiares, cuidadores e indivíduos com Síndrome de Down

TORQUATO, J. A. et al. A aquisição da motricidade em crianças portadoras de Síndrome de Down que realizam fisioterapia ou praticam equoterapia. Fisioter. Mov. v. 26, n. 3, p. 515-524, Jul./Set. 2013.

VASCONCELOS, A. C. C. P.; PEREIRA, I. D. F.; CRUZ, P. J. S. C. Práticas educativas em nutrição na Atenção Básica em Saúde: reflexões a partir de uma experiência de extensão popular em João Pessoa-Paraíba. Rev APS, v. 11, n. 3, p. 334-340, Jul./Set. 2008.

VOIVODIC, M. A. M. A.; STORER, M. R. S. O desenvolvimento cognitivo das crianças com síndrome de Down à luz das relações familiares. Psicologia: teoria e prática, v. 4, n. 2, p. 31-40, Jul./Ago. 2002. 